\title{
Atención a la Diversidad Cultural del Alumnado: Un Recorrido por Leyes de Educación de Ámbito Español
}

\author{
Attention to the Cultural Diversity of the Student: A Travel \\ through the Laws of Spanish Education
}

\section{Atenção a Diversidade Cultural do aluno: Uma Viagem pelas Leis da Educação Espanhola}

\author{
María Verdeja * \\ Universidad de Oviedo
}

\begin{abstract}
Desde el punto de vista político, la atención a la diversidad cultural del alumnado es uno de los retos de las sociedades del siglo XXI. Realizamos un recorrido por las diferentes leyes de educación en el ámbito español comenzando nuestro recorrido por la LGE y terminando el mismo con la LOMCE. El propósito de realizar tal recorrido es ofrecer una sintética perspectiva histórica que nos permita tomar consciencia del carácter ideológico y político de la educación al que Freire (19211997) en tantas ocasiones hizo referencia. Me propongo realizar una revisión de la política educativa que ha tenido lugar en nuestro país con el objetivo de analizar el tratamiento de la atención a la diversidad cultural del alumnado mencionando, también, aspectos ideológicos y retos educativos del momento.
\end{abstract}

Descriptores: Política, Diversidad cultural, Justicia social, Legislación educacional.

From the political point of view, attention to the cultural diversity of students is one of the challenges of $21^{\text {st }}$ century societies. We do a tour of the different laws of education in the Spanish sphere starting our journey through the LGE and ending the same with the LOMCE. The purpose of this tour is to offer a synthetic historical perspective that allows us to become aware of the ideological and political character of the education that Freire (1921-1997) referred to so many times. I propose to carry out a review of the educational policy that has taken place in our country with the objective of analyzing the treatment of the attention to the cultural diversity of students, also mentioning ideological aspects and educational challenges of the moment.

Keywords: Politics, Cultural diversity, Social justice, Educational legislation.

Do ponto de vista político, a atenção à diversidade cultural dos alunos é um dos desafios do século xxi. Abordamos as diferentes leis da educação no contexto Espanhol começando o nosso percurso pela lge e terminando com o lomce. $\mathrm{O}$ objectivo deste percurso é fornecer uma perspectiva histórica e sintética que permita tomar consciência da educação política e ideológica que Freire (1921-1997) tantas vezes referiu. Procuro proceder a uma revisão da política educacional que ocorreu em nosso país, com o fim de analisar o tratamento e a atenção para a diversidade cultural dos ditos alunos, e dos aspectos ideológicos e dos actuais desafios educacionais.

Palavras-chave: Política, Diversidade cultural, Justiça social, Legislação educacional.

*Contacto: verdejamaria@uniovi.es

ISSN: 2254-3139

www.rinace.net/riejs/

revistas.uam.es/riejs
Recibido: $\quad 3$ de diciembre 2016

$1^{\text {a }}$ Evaluación: 15 de enero 2017

$2^{\text {a }}$ Evaluación: 19 de febrero 2017

Aceptado: $\quad 7$ de marzo 2017 


\section{Introducción}

Es preciso comenzar diciendo que las migraciones y la globalización han sido, entre otros, factores que han contribuido a la configuración de sociedades en la que habitan diferentes culturas, es decir sociedades multiculturales. Según la UNESCO (2006) la diversidad cultural representa una manifestación de la diversidad de la vida en la tierra en lo referente a la multiplicidad de formas en que se expresan las culturas de los grupos y sociedades. En el contexto socioeducativo actual el fenómeno de la multiculturalidad se destaca como una característica básica de nuestra sociedad. Este hecho también se releja en los contextos educativos actuales. Según datos del Ministerio de Educación, Cultura y Deporte (MECD, 2016, p. 8) en el curso escolar 2016-2017, en España hay un total de 715.409 estudiantes extranjeros.

La preocupación por la diversidad cultural ha llevado a que diversos autores planteen los retos educativos que conlleva educar en sociedades multiculturales (Pérez Tapias, 2010; Banks, 2014). La presencia de alumnado extranjero en la escuela fruto de procesos migratorios no representa un hecho reciente pero es ahora, en los últimos años, cuando más se ha incrementado (Braga y Hevia, 2009). En nuestra comunidad autónoma, Principado de Asturias, también se puede constatar la presencia de alumnado extranjero en la escuela (Grupo Eleuterio Quintanilla, 2003; Louzao y González Riaño, 2007; Braga y Hevia, 2009). La educación en contextos educativos multiculturales conlleva desarrollar actuaciones orientadas a la atención a la diversidad cultural del alumnado (Essomba, 2012; Fueyo y Hevia, 2012; Louzao, 2009).

La educación tiene naturaleza política en todo el mundo tal y como señala Freire (1994) y por tanto no podemos negar el carácter político de la educación ni tampoco su trasfondo ideológico. Las leyes de educación que han tenido lugar en nuestro país, comenzando por la Ley 14/1970, de 4 de agosto, General de Educación y Financiamiento de la Reforma (LGE) y finalizando con la Ley Orgánica 8/2013 para la Mejora de la Calidad Educativa (LOMCE), han tenido diferentes implicaciones educativas, sociales, cultuales y pedagógicas que ponen de manifiesto el carácter político de la educación.

La preocupación por atender a la diversidad del alumnado en el ámbito educativo empieza a calar en las políticas públicas europeas hacia los años 70 y se plasma de un modo patente en el Tratado de la Unión Europea de 1992. El Tratado de la Unión Europea o Tratado de Maastricht es, junto al Tratado de Funcionamiento de la Unión Europea, uno de los tratados fundacionales de la Unión Europea. Firmado en la ciudad neerlandesa de Maastricht el 7 de febrero de 1992, entró en vigor el 1 de noviembre de 1993 y fue concebido como la culminación política de un conjunto normativo, vinculante para todos los Estados miembro de la Unión Europea, tanto para los futuros miembros como para los estados firmantes en el momento del tratado.

En el ámbito legislativo español hay algunas leyes orgánicas como la Ley Orgánica 1/1990, de 3 de octubre, de Ordenación General del Sistema Educativo (LOGSE) donde aparecen esbozados principios de atención a la diversidad pero no es hasta la Ley Orgánica 2/2006, de 3 de mayo, de Educación (LOE) cuando se introducen referencias explícitas a tales principios de la Comisión Europea. 


\section{Breve recorrido histórico desde la LGE de1970 hasta la LOMCE de 2013}

En el contexto educativo español en el campo de las enseñanzas medias, las reformas educativas y contrarreformas han sido la tónica hasta nuestros días. Nos encontramos en uno de esos momentos de reforma educativa y prueba de ello es la aprobación el 9 de diciembre de 2013 de la Ley Orgánica 8/2013 para la Mejora de la Calidad Educativa (LOMCE). Este cambio legislativo conlleva modificaciones en la organización, estructura y currículo en las diferentes etapas educativas que configuran nuestro sistema educativo. Su aprobación modifica la Ley Orgánica 2/2006, de 3 de mayo de Educación (LOE) y una disposición adicional de la Ley Orgánica 8/1985, de 3 de julio reguladora del Derecho a la Educación (LODE).

Para abordar la revisión teórica en la legislación educativa -ámbito español- me propongo realizar un recorrido histórico por las leyes de educación que han tenido lugar en nuestro país poniendo la mirada en el tratamiento de la atención a la diversidad cultural del alumnado, mencionando, también, otros aspectos ideológicos de las mismas. Los cambios en la legislación educativa, tal y como señala Freire (1994), obedecen a cuestiones ideológicas, sociales y económicas. Por este motivo considero importante hacer un recorrido que nos permita tener una perspectiva histórica pero que también nos ayude a comprender el contexto sociopolítico en el que tienen lugar determinados fenómenos educativos.

\subsection{Ley 14/1970, de 4 de agosto, General de Educación y Financiamiento de la Reforma Educativa (LGE)}

La sociedad española de los años 60 y 70 se caracterizó por el desarrollo económico y por el cambio social. Consecuencia de ello fueron los grandes movimientos migratorios desde las zonas rurales a las ciudades con efectos tanto para unas como para otras. A nivel educativo el panorama español se podría definir como un sistema con importantes carencias, masificación, fracaso escolar elevado y desescolarización de sectores minoritarios marginados

Comenzamos nuestro recorrido con la Ley 14/1970, de 4 de agosto, General de Educación y Financiamiento de la Reforma Educativa (LGE). Dicha ley que estuvo vigente -aunque de forma parcial- hasta 1990, fue impulsada por el Ministro de Educación José Luis Villar Palasí. La gran aportación de esta ley fue la creación de una educación general básica, única y obligatoria hasta los catorce años. En una primera fase se cursaba la Educación General Básica (EGB) y tras esta primera fase de ocho cursos el alumnado accedía al BUP (Bachillerato Unificado Polivalente), o a la recién implantada FP (Formación Profesional).

Con esta ley (LGE) se reformó todo el sistema educativo, desde la educación preescolar hasta la universitaria, adaptándolo a las necesidades del momento. Autores como Lerena (1976) critican que la LGE estaba diseñada con una profunda jerarquización interna de la población escolar, lo que a vez respondía a la jerarquización de las diferentes posiciones de partida. Recordamos que el contexto sociopolítico en el que se redacta la LGE es en el marco de la dictadura franquista. El sistema educativo español necesitaba una profunda reforma para estar más equiparado al entorno europeo. El objetivo de la LGE había sido dotar a España de un sistema educativo más equitativo y de mayor calidad. 
En relación con la atención a la diversidad del alumnado no encontramos, en dicha ley, actuaciones concretas. A pesar de esta observación, el texto enunciaba que: La Educación General Básica tiene por finalidad proporcionar una formación integral, fundamentalmente igual para todos y adaptada, en lo posible, a las aptitudes y capacidades de cada uno. Sin embargo, a pesar de tal afirmación, no se aprecian medidas específicas para atender a la diversidad del alumnado.

\subsection{Ley Orgánica 5/1980, de 19 de junio, por la que se regula el Estatuto de Centros Escolares (LOECE, 1980)}

Ley Orgánica 5/1980, de 19 de junio, por la que se regula el Estatuto de Centros Escolares (LOECE) fue una ley elaborada por el grupo político de Unión de Centro Democrático (UCD) y publicada en el BOE en 1980. Trataba de desarrollar el Artículo 27 -referido al Derecho a la Educación- reconocido en la Constitución Española (1978) con una perspectiva conservadora.

El grupo político del Partido Socialista Obrero Español (PSOE) la recurrió ante el Tribunal Constitucional por no respetar el espíritu y la letra de nuestra Constitución, quien le dio la razón en bastantes de sus objeciones. La sentencia dictada por el Tribunal Constitucional (Sentencia 5/1981 de 13 de febrero de 1981) fue -y sigue siendoimportante para fijar los límites de las libertades en la enseñanza y el respeto al derecho a la educación. Dicha ley (LOECE) nunca llego a aplicarse.

En 1982 y tras un estrafalario intento de golpe de Estado -el 23 de febrero de 1981- el grupo político del PSOE gana democráticamente las elecciones y llega a la presidencia del gobierno. Aunque ya se habían ido derogando algunos artículos de la LGE, aún seguían en vigor sus aspectos fundamentales, además, dicha ley, había sido objeto de duras críticas por parte de la izquierda, entre otras razones, porque era herencia de la dictadura franquista. De ahí la urgencia del nuevo gobierno por cambiarla. En España, aunque el marco legal se encontraba en la Ley General de Educación de 1970, la educación compensatoria se desarrollaba según el Real Decreto 27 de abril de 1983, publicado en el BOE del 11 de mayo, con la creación de un programa específico para beneficiar a las zonas geográficas o grupos de población que, por sus especiales características, requerían una atención educativa preferente.

\subsection{Ley Orgánica 8/1985 reguladora del Derecho a la Educación (LODE, 1985)}

El primer Ministro de Educación del Gobierno Socialista fue el profesor José María Maravall, bajo cuya dirección e impulso se aprobaron leyes, tan importantes, como la Ley Orgánica 8/1985 reguladora del Derecho a la Educación LODE. Como aspecto destacable de esta ley (LODE) podemos decir que incorporó, entre otras cosas, el sistema de conciertos, garantizando, así, el derecho de las familias a la libertad de elección de centro. La nueva ley (LODE) subraya de manera especial la importancia de la participación de la comunidad educativa, regulando el funcionamiento de los Consejos Escolares. En su preámbulo se plantea la participación como garantía de libertad y de influencia de los agentes sociales en la vida de los centros.

Como nos recuerda Leiva (2012) dicha ley (LODE) pasará a la historia como la primera ley orgánica que recoge en su primer capítulo el derecho de todos los españoles y de los extranjeros residentes en España a recibir una educación básica, obligatoria y gratuita aunque es conveniente recordar que, este derecho, estaba restringido a los legalmente residentes en España. En el artículo 20 de la misma se indica que, en ningún caso, se 
hará discriminación en la admisión de alumnado por razones ideológicas, religiosas, morales, sociales, de raza o nacimiento.

En este contexto, empiezan a colaborar con el Ministerio de Educación y Ciencia (MEC) algunos profesores descritos por Santos Arrabal (2013, p. 20) "como inteligentes y competentes que, con las ideas más claras sobre lo que había que hacer y con una buena dosis de formación, promueven un importante cambio".

\subsection{Ley Orgánica 1/1990, de 3 de octubre, de Ordenación General del Sistema Educativo (LOGSE, 1990)}

El equipo no llegaría a configurarse hasta la llegada al MEC, en 1988, de Javier Solana y de dos personas clave para la tarea reformadora: el Secretario de Estado de Educación, Alfredo Pérez Rubalcaba y el Director General de Ordenación Académica, Álvaro Marchesi. Bajo su dirección se produciría un cambio realmente radical en el panorama educativo español (Santos Arrabal, 2013).

El nuevo equipo ministerial es constructivista e inspirado en los Movimientos de Renovación Pedagógica (MRP). Autores como Hernández Díaz(2011) señalan que la LOGSE (1990) difícilmente hubiera sido posible sin el proceso de debate que le precedió, inserto en los procesos de participación y discusión de los MRP.

Con esta mentalidad y con estos criterios se escribe y aprueba la Ley Orgánica 1/1990, de 3 de octubre, de Ordenación General del Sistema Educativo, (LOGSE) cuya implementación escolar se iniciaría dos años más tarde. Dicha ley fue aprobada por todos los grupos parlamentarios excepto por el grupo del Partido Popular (precisamente el partido que accediendo a la presidencia del gobierno en el año 1996, tendría que encargarse de aplicar la misma). Entre otras medidas, amplió la escolaridad obligatoria hasta los 16 años. También contemplaba iniciativas tales como como un nuevo currículo que otorgaba a los equipos docentes una mayor capacidad de decisión y autonomía.

Además, se subrayaba la importancia de tener en cuenta las situaciones en las que se encuentren los estudiantes y, desde tal planteamiento, se aboga por un modelo de centro educativo abierto a la diversidad y al entorno social, que fuese cooperativo e integrado con el contexto socioeducativo. Asimismo, se consideraba como principio básico el rechazo de todo tipo de discriminación y se apuesta por un modelo educativo que respete a todas las culturas. También se destaca la necesidad de desarrollar la capacidad creativa, los hábitos de comportamiento democrático y espíritu crítico del alumnado.

Al ser aprobada la LOGSE queda derogada la Ley General de Educación (LGE). Con la aprobación de esta ley (LOGSE) se contemplan, por vez primera, las medidas específicas para compensar las desigualdades derivadas de situaciones sociales, laborales y económicas de toda índole, pero no se hace referencia directa -o específica- a los estudiantes extranjeros. Esto, según indica Leiva (2012), está motivado porque la llegada masiva de los mismos se producirá a lo largo del decenio de los noventa.

Si la atención al alumnado con necesidades de compensación educativa se rige por los principios de normalización e integración favoreciendo que, en la medida de lo posible, sean escolarizados en un entorno lo más normalizado posible, la LOGSE en su artículo 37 también añade que, la escolarización en centros específicos solamente se hará cuando el centro ordinario no disponga de los recursos adecuados para poder dar respuesta a las necesidades específicas que presente el alumnado. 
En la LOGSE, no se diferencia -de forma específica- el tratamiento de los estudiantes de origen extranjero de otros que, por diversos factores, tienen dificultades de aprendizaje y que han de recibir una atención personalizada. Lo que si resalta, en su preámbulo, es la importancia que se le concede a la educación para avanzar en la lucha contra la discriminación y desigualdad por cualquier tipo de razón (raza, sexo, clase social, etc.) Es necesario tener en cuenta que la LOGSE se redactara antes del espectacular crecimiento de la inmigración de origen extranjero y también es conveniente recordar que en aquellos momentos la mayor preocupación educativa tenía que ver con la atención a estudiantes con necedades educativas especiales.

La LOGSE introduce con claridad el concepto de necesidades educativas especiales incluyendo en este grupo a los estudiantes que, por encontrarse en situaciones sociales o culturalmente desfavorecidas, requieren durante su escolarización la prestación de apoyos y actuaciones educativas específicas. Esto significó un paso adelante que se continuó con el Real Decreto 299/1996 de 28 de febrero de ordenación de las acciones dirigidas a la compensación de desigualdades en educación y que tiene como objetivo regular las medidas que permitan prevenir y compensar las desiguales en educación derivadas de factores sociales, económicos, culturales, geográficos, étnicos o de cualquier tipo.

\subsection{Ley Orgánica 9/1995, de 20 de noviembre, de la Participación, la Evaluación y el Gobierno de los Centros docentes (LOPEGCE)}

Ley Orgánica 9/1995, de 20 de noviembre, de la participación, la evaluación y el gobierno de los centros docentes es conocida, también, como la "Ley Pertierra" por ser Gustavo Suárez Pertierra, Ministro de Educación de la corporación Socialista, quien la firmó. Podemos decir que fue una ley centrada en la gestión y gobierno de los centros docentes y en ese sentido, dicha ley, ha regido cómo debe ser la participación y gestión de los centros educativos, ampliando los ámbitos de la participación de los distintos sectores de la comunidad escolar y la autonomía de los centros para desarrollarla. Cobran así importancia los Consejos Escolares de los centros adquiriendo estos, entre otras competencias, capacidad para elegir a los directores/as de los centros -capacidad que quedaría sistematizada en la citada ley (LOPEGCE) en detrimento de los Claustros de profesores-. La LOPEGCE continuó con más planteamientos de educación compensatoria ya que esta ley incluía medidas específicas para compensar desigualdades derivadas de situaciones sociales y económicas desfavorecidas.

Concretamente -Disposición Adicional Segunda- se establecía que las administraciones educativas tenían que garantizar la escolarización del alumnado con necesidades educativas especiales, manteniendo una distribución equilibrada de los mismos, considerando su número y sus especiales circunstancias de manera que se desarrolle eficazmente la idea integradora.

Se entendía por alumnado con necesidades educativas especiales aquel alumnado que, por tener algún tipo de discapacidad, por padecer trastornos graves de la conducta o por estar en situación social o cultural desfavorecida, precisaban de una atención educativa específica por parte del centro.

Destacamos como especialmente relevante el dato que incorporaba esta ley en lo referente a la distribución equilibrada del alumnado con necesidades educativas ya que, como sabemos, la realidad muestra que actualmente no existe tal equilibrio, sino que 
como indican algunos estudios (Louzao, 2009; Louzao y González Riaño, 2007) la mayor parte de este alumnado tiende a estar escolarizado en la escuela pública, fenómeno que también se da en relación con el alumnado extranjero.

Tenemos, por tanto, que las administraciones educativas reconocían abiertamente la obligación de garantizar los recursos adecuados para que todo el alumnado con necesidades educativas especiales alcance los mismo objetivos generales que el resto del alumnado y para ello pone en marcha una política educativa compensatoria, dotando a los centros de los recursos necesarios. Estas medidas fueron desarrolladas por el Real Decreto 299/1996, que recoge los recursos para la educación compensatoria y que sigue teniendo vigencia en la actualidad. Entre sus disposiciones cabe desatacar la formación del profesorado, facilitar el acceso y la permanencia en el sistema educativo, y otro tipo de medidas educativas como la regulación del profesorado de educación compensatoria, con un perfil de Pedagogía Terapéutica.

Posteriormente la Orden Ministerial del 22 de Julio de 1999 recogía las acciones de compensación educativa a desarrollar en los centros sostenidos con fondos públicos. Con esta orden se proporcionaron los recursos necesarios, tanto materiales como humanos, a aquellos centros que escolarizaban alumnado con necesidades de compensación educativa entendiendo por este concepto a aquel alumnado que, por su pertenencia a minorías étnicas o culturales en situación de desventaja socio-educativa, podía necesitar algún tipo de apoyo específico.

También se contemplaba al alumnado que, por su pertenencia a un grupo socialmente desfavorecido, evidencia un desfase curricular significativo, con una diferencia de dos o más años entre su nivel de competencia curricular y el nivel en el que está escolarizado, presentando además otros tipo de necesidades afectivas y de apoyo debido a circunstancias tales como una escolarización tardía, irregular o, en el caso del alumnado extranjero, por desconocimiento de la lengua vehicular del proceso de enseñanza aprendizaje.

\subsection{Ley Orgánica 10/2002, de 23 de diciembre, de Calidad de la Educación (LOCE, 2002)}

En el año 1996, en pleno proceso acelerado de implantación de la LOGSE gana las elecciones el Partido Popular bajo la presidencia de José María Aznar. La relación de los Populares con la LOGSE fue deteriorándose cada vez más y tras su llegada al poder, con el nombramiento de Esperanza Aguirre como Ministra de Educación y Cultura, se emprendió una campaña para cambiar esta ley.

El Gobierno del Partido Popular, tenía como principal objetivo cambiar cuanto antes la LOGSE, pero el desarrollo normativo de la nueva propuesta de ley formulada por el Partido Popular (LOCE) resultó más lento de lo esperado y la nueva ley no vio la luz en el BOE hasta el 24 de diciembre de 2002 -seis años después de su triunfo electoral-. Sus desarrollos normativos todavía tardarían en publicarse y el proceso estaba siendo lento teniendo en cuenta que su calendario de implantación estaba previsto para el curso 2004/05-. Ante esta situación, y contra todo pronóstico, en el proceso electoral del año 2004 el Partido Popular pierde las elecciones ganando las mismas el Partido Socialista Obrero Español (PSOE) quien, como refiere Santos Arrabal (2013, p. 24) no estaba dispuesto a permitir que se hiciera efectiva una ley de educación (LOCE) que había nacido contra la ley estrella de la izquierda (LOGSE). 
Así que, tras la llegada a la presidencia del gobierno el Partido Socialista Obrero Español (PSOE) teniendo como Presidente a José Luis Rodríguez Zapatero, se paralizó el calendario de aplicación de la citada ley (LOCE) por medio del Real Decreto 1318/2004 de 28 de mayo de 2004 y dicha ley nunca llegó a aplicarse. Con relación a la atención a la diversidad del alumnado podemos decir que la LOCE contemplaba la adopción de medidas educativas discriminatorias hacia los hijos e hijas de los inmigrantes, a los que preveía escolarizar en aulas específicas de modo exclusivo o en programas de aprendizaje profesional convirtiendo, con tales medidas, el sistema educativo en un instrumento de desigualdad, marginación y segregación.

\subsection{Ley Orgánica 2/2006, de 3 de mayo, de Educación (LOE, 2006)}

La nueva Administración socialista abre con carácter inmediato un debate sobre la reforma educativa. Como describe Santos Arrabal (2013 p. 25), la LOE (2006) avanza simultáneamente con rapidez bajo la dirección de Alejandro Tiana. La Ley Orgánica 2/2006, de 3 de mayo, de Educación se publica en el BOE el día 4 de mayo del año 2006, apenas dos años después de haberse suspendido el calendario de implantación de la LOCE manteniéndose vigente, hasta entonces, la LOGSE. Para autores como Santos Arrabal (2013) la nueva ley (LOE) supone una mejora, entre otras cosas, porque engloba y perfecciona aspectos contenidos en otras leyes educativas. El ejecutivo de José Luis Rodríguez Zapatero elaboró la LOE que permanece vigente desde el año 2006 hasta el curso académico 2013/2014.

También se hace necesario tener en cuenta el contexto sociopolítico del país así pues, la LOE se redacta en un momento en el proliferan propuestas de cambio relacionadas con la ciudadanía y en los que la diversidad cobra una especial importancia (legalización del matrimonio homosexual, medidas de protección integral contra la violencia de género, la ley de dependencia, el reconocimiento de la plurinacionalidad del Estado).

Entre otras observaciones podemos destacar, como novedad, la introducción de una nueva materia en el currículo: "Educación para la Ciudadanía y los Derechos Humano". Dicha materia desde la aprobación de la referida LOE no ha estado exenta de polémica, pese a que desde diversos ámbitos internacionales y europeos (UNESCO, 2010; Consejo de Europa, 2008) se instaba a los gobiernos a llevar a cabo iniciativas relacionadas con la Educación para la Ciudadanía y los Derechos Humanos.

Con la promulgación de la LOE se da un paso hacia adelante en el campo de la atención a la diversidad. Una muestra de ello es que en su preámbulo recoge la necesidad de que exista un compromiso de toda la comunidad educativa y de todos los centros para logar una adecuada atención a la diversidad. Otro ejemplo, lo encontramos en el Artículo 2, referente a los fines de la educación, donde en el apartado g) se hace referencia a la formación en el respeto y reconocimiento de la pluralidad lingüística y cultural de España y de la diversidad cultural como un elemento enriquecedor de la sociedad. Cabe destacar que la LOE considera la atención a la diversidad del alumnado como una de las finalidades educativas fundamentales atendiendo a los principios de inclusión y no discriminación y, desde estos planteamientos, sienta la bases para que las distintas administraciones educativas lleven a cabo esta finalidad propuesta en dicha ley y desarrollen actuaciones encaminadas a hacerla realidad.

Dentro de la LOE se contemplan medidas específicas relacionadas con la incorporación tardía del alumnado extranjero, por ejemplo, cuando dicha situación -la incorporación 
tardía- supone dificultades para seguir el currículo, bien por desconocimiento del idioma (español/castellano, catalán/valenciano, euskera y gallego) como lenguas del proceso de enseñanza-aprendizaje o bien por desconocimiento de unas competencias de aprendizajes básicos. Además, en uno de sus apartados -el Título II, referido al alumnado con necesidad específica de apoyo educativo- contempla la consideración de la situación del alumnado de incorporación tardía y reconoce que es necesario, para dicho alumnado, poner en marcha una serie de medidas educativas para favorecer que su proceso de integración sea efectivo y pueda acceder y promocionar en el sistema educativo en las mismas condiciones de igualdad que el resto del alumnado.

El marco normativo de la LOE es favorecedor del desarrollo práctico de la atención a la diversidad cultural en la escuela; no solo porque implica el respeto y aprovechamiento de la diversidad cultural sino porque, además, posibilita el planteamiento de focalizar una cultura de la diversidad en la escuela; lo que significa ir más allá de la multiculturalidad en términos de conocimiento cultural. Tales planteamientos están en coherencia con los de la UNESCO (2006) en cuanto que se entiende que la educación intercultural se propone ir más allá de la coexistencia pasiva, y se apuesta por lograr un modo de convivencia evolutivo y sostenible en sociedades multiculturales, propiciando la instauración del conocimiento mutuo, el respeto y el diálogo entre los diferentes grupos culturales.

Con relación a la etapa de la Educación Secundaria Obligatoria, la Ley Orgánica de Educación (LOE) pone el énfasis en la atención a la diversidad del alumnado y en la prevención de las dificultades de aprendizaje actuando tan pronto se detecten. Para ello los centros docentes considerarán, de forma prioritaria, las medidas de atención a la diversidad a aplicar al alumnado teniendo en cuenta la diversidad: alumnado que presente necesidades educativas especiales, alumnado con altas capacidades intelectuales, alumnado con integración tardía al sistema educativo español y alumnado en desventaja educativa por motivos socioculturales o socioeconómicos.

\subsection{Ley Orgánica $8 / 2013$, de 9 de diciembre, para la Mejora de la Calidad Educativa (LOMCE)}

El 20 de noviembre de 2011 y tras la convocatoria anticipada de elecciones gana las mismas el grupo del Partido Popular llegando a ocupar la presidencia del gobierno Mariano Rajoy. Con la llegada del nuevo gobierno al poder, nuevamente, volvemos a un cambio en la legislación educativa. Es durante el mandado del Partico Popular cuando se acomete una nueva reforma educativa y se promulga la Ley Orgánica 8/2013, de 9 de diciembre, para la mejora de la calidad educativa (LOMCE). Para algunos autores (Bolívar y San Fabián, 2013) la actual reforma educativa (LOMCE) emprendida por el grupo político del Partido Popular, supone el mayor cambio educativo desde la Ley General de Educación (LGE).

Desde la presentación del proyecto de ley el 17 de mayo de 2013 por el ministro de Educación, Cultura y Deporte del Partido Popular, José Ignacio Wert, hasta su aprobación definitiva por las Cortes Generales de España el 28 de noviembre de 2013, ha sido una ley controvertida que ha despertado numerosas protestas por sectores sociales y políticos. Una muestra de ello es que durante su tramitación en las Cortes, dicha ley (LOMCE) no fue apoyada por ningún otro grupo parlamentario que el Partido Popular. Dicha ley, ha sido aprobada gracias a la mayoría absoluta del grupo parlamentario que la ha promulgado, es decir: el grupo político del Partido Popular, y se ha llevado a cabo sin contar con el apoyo de ninguna otra corporación política y con escaso respaldo de la 
ciudadanía o de la propia comunidad educativa. Cabe señalar que el contexto sociopolítico en el que se redacta la LOMCE es un momento en el que adquiere fuerza la ideología neoliberal y ello conlleva, entre otras cosas, el rechazo de políticas sociales y educativas con una importante base comunitaria y favorecedora de la diversidad cultural.

La Ley Orgánica 8/2013, de 9 de diciembre, para la mejora de la calidad educativa denominada popularmente como "Ley Wert", es una ley del ordenamiento jurídico español con carácter de Ley Orgánica que modifica la Ley Orgánica 2/2006, de 3 de mayo, de Educación (LOE, 2006) y seis artículos y una disposición adicional de la Ley Orgánica 8/1985, de 3 de julio, reguladora del Derecho a la Educación (LODE).

Paulo Freire (citado en Gadotti, 2007, p. 34) plantea que "las reformas pedagógicas para el pueblo fracasan porque se hacen para él y no por él”, es decir, el pueblo termina siendo el objeto y no el sujeto activo de los cambios. Desde esta perspectiva, podemos inferir que esto mismo sucede con la aprobación de la LOMCE puesto que ha sido una reforma educativa impuesta por el gobierno, que gozaba de mayoría absoluta, pero sin contar con el consenso de la ciudadanía ni de otros grupos políticos.

Tras la aprobación de la LOMCE el 9 de diciembre de 2013, se elimina del currículo la materia de Educación para la Ciudadanía y los Derechos Humanos, que en la LOE tenía carácter obligatorio, y aparecen nuevas materias como: Valores Culturales y Sociales en Primaria y Valores Éticos en Secundaria, que constituyen una alternativa a la de Religión. Asimismo, entre otras novedades que incorpora la ley (LOMCE) señalamos la de otorgar mayor autonomía para los centros y la de potenciar la figura del director atribuyéndole más competencias a la hora de tomar decisiones en detrimento de las funciones que venía desempeñando el Consejo Escolar.

Por lo que respecta a la atención a la diversidad del alumnado podemos decir que la LOMCE pese a mantener algunas iniciativas y programas que se venían desarrollando anteriormente y favorecer la autonomía organizativa y curricular de los centros, no introduce medidas específicas a este respecto. Sin embargo, se apuesta por la introducción de itinerarios formativo-profesionales comenzando éstos a los 13 años.

En relación con esta medida el Consejo de Estado (2013) también se ha pronunciado señalando la existencia de otros informes (OECD, 2012) que abogan por evitar la separación temprana y posponer la selección de estudiantes hasta la educación media o superior y por lo tanto el adelanto de la elección de itinerarios en la ESO es una medida que se entiende que contribuirá a segregar de manera temprana al alumnado, como señala el citado informe. Asimismo hay autores autores (San Fabián y Braga, 2015) quienes también han criticado esta medida. Por otra parte, la investigación educativa desarrollada en el contexto del Proyecto INCLUD-ED sobre "Actuaciones de éxito en las escuelas europeas" (CREADE, 2011) establece que la separación en itinerarios antes de los 16 años genera desigualdades que se amplían cuanto antes se realizan. 


\section{Algunas cuestiones para reflexionar}

A pesar de las reformas educativas que han tenido lugar en nuestro país durante los últimos 40 años, cabe preguntarse en qué medida la etapa educativa de la secundaria obligatoria guarda, todavía, más relación con sus orígenes (véase el Real Decreto Plan General de estudios del 17 de septiembre de 1845) que con los derivados de las sucesivas reformas educativas. El planteamiento educativo del citado Real Decreto entendía que la enseñanza secundaria pretendía servir a las clases medias para ocupar un puesto preponderante y preparar a los estudiantes para la universidad. Es pues, el origen de la enseñanza secundaria -Plan Pidal de 1845- y su concepción dentro del sistema educativo español lo que, quizás, entraña una de las cuestiones más relevantes.

Resulta clave pensar que existen determinados fenómenos que se producen a lo largo de periodos que se dilatan en el tiempo. Teniendo en cuenta esto, autores como Viñao (2002) hacen referencia a que el proceso socioeducativo iniciado -según los países- en los últimos años del siglo XIX y los primeros decenios del siglo XX es un proceso que todavía ni está resuelto ni ha concluido. Es decir, a pesar de que las ideas pedagógicas que inspiraban reformas educativas promovidas por leyes orgánicas (LOGSE, LOE) y que tenían que ver con un modelo de enseñanza secundaria comprensiva, cabe pensar que en la actualidad todavía no hemos alcanzado tal propósito.

Sin embargo, recuerda Gimeno (2005), la educación secundaria obligatoria es una experiencia universal que caracteriza a las sociedades modernas como si se tratase de un rasgo antropológico de las mismas. Es, pues, un proyecto social y educativo que ha sido reconocido como un derecho universal porque encierra la posibilidad de dignificar a los seres humanos al tiempo que contribuye a la mejora de la sociedad. No podemos olvidar -aunque, tal vez, ya lo hemos hecho- su espíritu educativo y social.

Una muestra de que las reformas educativas no han logrado un modelo de enseñanza verdaderamente comprensiva -ni tampoco social- para todos los estudiantes, puede verse reflejado en las altas tasas de fracaso y abandono escolar que siguen teniendo lugar en nuestro país, como así lo refleja la investigación educativa (Bolívar y López, 2009; González Gozález, 2006).

Como señalan Bolívar y López (2009, p. 57) cuando la LOGSE diseñó el currículum y estructura de la Educación Secundaria se planteaba que un 90\% de los estudiantes debía lograr el título de Graduado de ESO. Sin embargo, tal objetivo, no se ha logrado y en la actualidad uno de cada tres estudiantes no termina la ESO, es decir; no obtiene la titulación de graduado en Educación Secundaria Obligatoria. Por otra parte, diversos organismos europeos y nacionales (Comisión Europea, 2008; MECD, 2016) también señalan esta problemática y nos alertan acerca de la necesidad de reducir las tasas de abandono y fracaso escolar.

Esto nos lleva a pensar en cuestiones tan importantes como la igualdad de oportunidades, la justicia social, el carácter público y popular de la educación, la defensa y promoción de los que menos tienen, la lucha contra todo tipo de discriminación (Verdeja, 2015).

Desde algunos planteamientos (Lucio-Villegas, 2015; Verdeja, 2015) para Freire la educación representa un instrumento al servicio de la justicia social. Plantearse el tema de la justicia social y de igualdad de oportunidades en el sistema educativo, recuerda 
Torres Santomé (2008), pasa por analizar y evaluar el grado en que el currículo escolar es respetuoso con las distintas idiosincrasias de los colectivos y personas que tienen que convivir en esa institución, una escuela y un contexto educativo que, recordamos, es multicultural.

Por otra parte, con relación a la igualdad de oportunidades, Fernández Enguita (1987, p. 76) menciona que las reformas comprensivas tratan de responder a una cierta conciencia social sobre la necesidad de la igualdad, formulando esta como igualdad de resultados y de oportunidades. Cabe preguntase en qué medida tales reformas han contribuido, realmente, a fomentar tal igualdad de oportunidades.

Como señala San Fabián (2011) esta sucesión de leyes puede entenderse en parte por los cambios que ha experimentado la sociedad pero, también en parte por razones de oportunidad política donde juega un papel importante el poder y el control:

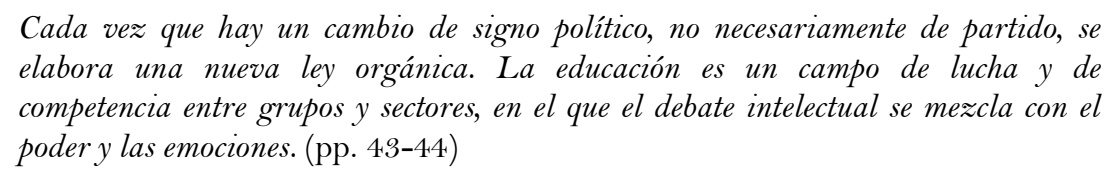

Para autores como Gimeno (citado en San Fabián, 2011, p 44) esta proliferación de leyes es en parte consecuencia de la falta de un consenso entre las fuerzas políticas sobre las funciones esenciales del sistema educativo, sobre los fines de la educación, sobre lo que debe contener la escolaridad mínima común para todos y sobre las grandes líneas de organización del sistema (regulación pública-privada, gobierno de los centros, participación...).

Además, destaca San Fabián (2011) que, a pesar de que existen diferencias entre unas leyes y otras, todas carecen de un diagnóstico preciso acerca del estado del sistema educativo y ninguna ha sido evaluada y además destaca: "Su corta vida provoca un arraigo superficial y crea frustración y resistencia entre los docentes, que adquieren, eso sí, un nuevo argot” (p. 45).

Las leyes educativas, en efecto, tienen un componente ideológico que, sin duda alguna, tiene gran repercusión en la configuración de los sistemas educativos. Dado que la educación tiene fuertes componentes socio-políticos, Freire (1994) alude a la imposibilidad de considerar la práctica educativa como una actividad neutra. No es posible, por tanto, asumir la educación como una actividad que pueda ser abordada exclusivamente desde una dimensión meramente técnica y metodológica -y por tanto neutra- puesto que tiene componentes sociales, económicos, políticos y culturales, etc.

Partiendo de la base de que la educación no es neutral se revela, por tanto, la dimensión política e ideológica de la educación. Si queremos que haya cambios, es necesario que exista voluntad política de cambio. Tal vez, uno de los grandes retos políticos y educativos del momento consista en entender el sentido educativo y social de la enseñanza secundaria y desarrollar un modelo de enseñanza comprensiva que tenga en cuenta los diferentes ritmos de aprendizaje, expectativas, motivación y necesidades de un alumnado diverso (Verdeja, 2015). 


\section{Algunas ideas pensando en la educación del siglo XXI}

En este contexto sociopolítico presentamos algunas ideas pensadas para ofrecer a los estudiantes una educación de calidad en el siglo XXI. Tales propuestas están inspiradas en la pedagogía de Paulo Freire y planteadas con lo que, considero, debe ser un modelo de escuela multicultural:

- Un modelo de escuela multicultural es una escuela permanentemente abierta al cambio y a la mejora. Ha de ser un modelo de escuela flexible, democrática, inclusiva y que, como señala Freire, reconoce la diferencia como un valor.

- Una escuela multicultural requiere operar en términos de humanidad y superar los etnocentrismos más primarios. Es necesario cuestionar aspectos asociales de nuestra cultura y fomentar el respeto por otras personas y su cultura.

- Es necesario re-plantear el modelo de formación y organización del profesorado, puesto que una escuela multicultural, necesariamente ha de contar con profesorado sensible ante otras culturas y que pueda enseñar y valorar la lengua materna de los estudiantes.

- La educación intercultural, de acuerdo con Freire, implica un proyecto pedagógico claramente orientado a favorecer el cambio social, entendido éste en el sentido de buscar principios de justicia social y una ética fundada en el derecho a las diferencias.

- La educación intercultural implica la necesidad de reflexionar acerca de injusticias y el sufrimiento (inmigración, pobreza, violencia contra las mujeres, etc.). Esto es, en esencia, lo que Freire entiende como educación problematizadora.

- Una escuela multicultural ha de estar abierta a la comunidad educativa y debe favorecer mecanismos de participación del profesorado, alumnado, familias y colectivos sociales. Es un modelo de escuela democrática que además de estar abierta permanentemente a la realidad contextual de los educandos -como soñaba Freire- debe estar dispuesta a aprender de sus relaciones con el medio.

- La educación intercultural conlleva necesariamente trabajar con diversidad de fuentes y recursos. Implica que el alumnado sea protagonista del proceso de enseñanza-aprendizaje y se convierta en sujeto activo de dicho proceso, utilizando recursos que despierten la motivación y el interés por aprender.

- Una escuela multicultural conlleva revisar la selección cultural de los contenidos que conforman el currículo común para hacerlo más universal y representativo de las culturas y de la realidad social. Al mismo tiempo, en la selección de los contenidos curriculares -como recuerda Freire- es necesario preguntarse por cuestiones tales como: ¿a quién pertenece esa cultura? o ¿a favor de quién está esa cultura? Cuestiones que nos servirán para comprender que, en definitiva, ninguna práctica educativa es neutra.

- El diálogo es una exigencia existencial, por ello Freire lo propone como método de conocimiento. También señala que tiene que producirse con respeto y atendiendo a los argumentos del otro. Una escuela multicultural debe favorecer 
que se produzcan procesos de comunicación dialógica que puedan modificar, en su caso, ideas erróneas o preconcebidas.

\section{Referencias}

Banks, J. A. (2014). Emigración global, diversidad y educación para la ciudadanía. Revista Electrónica Interuniversitaria de Formación del Profesorado, 17(2), 1-11.

Bolívar, A y López, L. (2009). Las grandes cifras del fracaso y los riesgos de exclusión educativa. Profesorado. Revista de currículum y formación del profesorado, 13(3), 51-78.

Bolívar, A. y San Fabián, J. L. (2013). La LOMCE, ¿una nueva ley para mejorar la calidad educativa? Organización y Gestión Educativa, 1, 7-11.

Braga, G. y Hevia, I. (2009). Una mirada hacia la infancia inmigrante en Asturias. Oviedo: Consejería de Bienestar Social y Vivienda.

Consejo de Europa. (2008). Libro blanco sobre el diálogo intercultural: Vivir juntos con igual dignidad. Estrasburgo: Consejo de Europa.

Consejo de Estado. (2013). Informe anteproyecto de ley orgánica de mejora de la calidad educativa. Madrid: Consejo de Estado.

Comisión Europea. (2008). Comunicación de la comisión al parlamento europeo, al consejo, al comité económico y social europeo y al comité de las regiones. Mejorar las competencias en el siglo XXI: Agenda para la cooperación europea en las escuelas. Bruselas: Comisión de Comunidades Europeas.

CREADE. (2011). Actuaciones de éxito de las escuelas europeas. Recuperado de: https://sede.educacion.gob.es/

Essomba, M. A. (2012). Inmigración, sociedad y educación en la UE. Hacia una política educativa de plena inclusión. Cultura E Educación, 24(2), 137-148. doi:10.1174/113564012804932074

Freire, P. (1994). La naturaleza política de la educación. Barcelona: Planeta De Agostini.

Fueyo, M. A. y Hevia, I. (2012). Educando para reconstruir las representaciones de los medios de comunicación y las industrias culturales sobre las personas emigrantes. Revista Electrónica Interuniversitaria de Formación del Profesorado, 15(2), 101-109.

Gadotti, M. (2007). La escuela y el maestro Paulo Freire y la pasión de enseñar. São Paulo: Publisher Brasil.

Gimeno, J. (2005). La Educación Secundaria Obligatoria: Su sentido educativo y Social. Madrid: Morata.

González González. M. T. (2006). Absentismo y abandono escolar: Una situación singular de la exclusión educativa. REICE. Revista electrónica iberoamericana sobre calidad, eficacia y cambio en educación, 4(1), 1-15.

Grupo Eleuterio Quintanilla. (2003). Alumnado extranjero en la escuela asturiana. Medidas urgentes para una educación de calidad. Recuperado de: http://www.equintanilla.com/

Hernández Díaz, J. M. (2011). La renovación pedagógica en España al final de la transición. Encuentro de los movimientos de renovación pedagógica y el ministro Maravall (1983). Revista d'Història de l'Educació, 18, 81-105.

OECD. (2012). Equity and quality in education. Supporting disadvantaged students and schools. París: OECD Publishing. 
Fernández Enguita, M. (1987). Enseñanza Secundaria y comprensividad. En VVAA., Seminario sobre el proyecto para la reforma de la enseñanza (pp. 75-84). Consejo Escolar de Estado. Madrid: Ministerio de Educación y Ciencia.

Leiva, J. J. (2012). Educación intercultural y convivencia en la escuela inclusiva. Málaga: Ediciones Aljibe S.L.

Lerena, C. (1976). Escuela, ideología y clases sociales en España crítica de la sociología empirista de la educación. Barcelona: Ariel.

Louzao, M. y González Riaño, X. A. (2007). La integración social y educativa del alumnado inmigrante en Asturias. Un estudio de Caso. En CIDE., Los premios nacionales de investigación educativa y tesis doctorales 2005 (pp. 11-41). Madrid: CIDE.

Louzao, M. (2009). Diseño, desarrollo y evaluación de un proyecto de educación intercultural en un centro asturiano de educación primaria. Una perspectiva de la investigación en la acción y el estudio de caso (Tesis doctoral). Oviedo: Universidad de Oviedo.

Lucio-Villegas, E. (2015). Paulo Freire. La educación como instrumento para la justicia social. Revista Internacional de Educación para la Justicia Social (RIEJS), 4(1), 9-20.

MECD. (2016). Datos y cifras: Curso escolar 2016-2017. Madrid: Ministerio de Educación, Cultura y Deporte.

Pérez Tapias, J. A. (2010). Educar desde la interculturalidad. Exigencias curriculares para el diálogo entre culturas. En J. Gimeno (Comp.), Saberes e incertidumbres sobre el currículum, (pp. 149-161). Madrid: Morata.

San Fabián, J. L. (2011). El papel de la organización escolar en el cambio educativo: La inercia de lo establecido. Revista de Educación, 356, 41-60.

San Fabián, J. L. y Braga, G. (2015). El derecho de la educación en Asturias. En P. Suárez y C. Becedóniz (Coords.), La infancia en Asturias 2015 (pp. 42-45). Gijón: UNICEF.

Santos Arrabal, M. (2013). El Pasado hasta casi el presente de los manuales escolares. En M. Beas (Ed.), Ciudadanías e identidades en los manuales escolares (1970-2012) (pp. 11-28). Sevilla: Diáda Editora.

Torres Santomé, J. (2008). Diversidad cultural y contenidos escolares. Revista de Educación, 345, 83-110.

UNESCO. (2006). Directrices de la UNESCO sobre la educación intercultural. París: UNESCO.

UNESCO. (2010). Invertir en la diversidad cultural y el diálogo intercultural. París: UNESCO.

Verdeja, M. (2015). Aportaciones de la pedagogía de Paulo Freire a la educación intercultural. Posibilidades de aplicación al sistema educativo en Asturias (Tesis doctoral). Universidad de Oviedo.

Viñao, A. (2002). Sistemas educativos, culturas escolares y reformas. Madrid: Morata.

\section{Breve CV de la autora}

\section{María Verdeja Muniz}

Licenciada y doctora en pedagogía por la Universidad de Oviedo. Actualmente trabaja como profesora asociada en el Departamento de Ciencias de la Educación, Área de Didáctica y Organización Escolar. En el ámbito universitario ha impartido docencia en diferentes Grados como el de Pedagogía y el de Educación Primaria. También ha impartido docencia en el Máster de Intervención e Investigación Socioeducativa. Fuera 
del ámbito universitario tiene más de 14 años de experiencia trabajando en el ámbito de la orientación laboral y la atención a colectivos en riesgo de exclusión social. Las temáticas de investigación que más le interesan tienen que ver con el estudio de la obra de Paulo Freire, la atención a la diversidad cultural del alumnado y la elaboración de materiales curriculares desde una perspectiva freireana. ORCID ID: 0000-0003-04999248. Email: verdejamaria@uniovi.es 